\title{
On the influence of electric power quality on the decimation of currents and voltages of utility frequency
}

\author{
Gennady Vagin ${ }^{1}$, Alexander Kulikov ${ }^{1}$, Alexander Sevostyanov ${ }^{1, *}$, and Anatoly Fitasov ${ }^{1}$ \\ ${ }^{1}$ Nizhny Novgorod State Technical University n.a. R.E. Alekseev, Minin str., 24, Nizhny Novgorod, Russia
}

\begin{abstract}
The electric power industry cannot develop without analyzing the factors that affect the shape of currents and voltages when determining the parameters of the electric network mode in the context of the introduction of modern means of intellectualization into the structure of power supply systems. The share of equipment affecting the electric power quality reaches $90 \%$ in modern electrical networks. This leads to the appearance of noise in the network that reduces the quality of electrical energy, voltage distortion by higher harmonic components in particular, and affects the adequacy of the parameters of the electrical network adopted by automatic control devices. The article presents the results of digital signal processing containing distortions in the form of a harmonic component with a decrease in the sampling frequency. It is shown that a decrease in the sampling frequency does not exclude the effect of higher harmonics on the result of processing signals, including high-frequency distortions.
\end{abstract}

\section{Introduction}

The electric power quality is one of the main factors affecting the trouble-free operation of automatic control devices in industrial power supply systems [1]. Nowadays these devices include components built based on power electronics and microprocessor technology. Incorrect operation of automatic control devices in the electrical network can be caused by the presence of electromagnetic disturbances in the form of deviations, fluctuations, or non-sinusoidal supply voltage.

It should be noted that electromagnetic disturbances generated by consumers' electrical receivers have a negative impact not only on consumer equipment but also on equipment installed in the supply distribution network. So, the appearance of non-sinusoidal current in the network can cause additional heating and malfunctioning of the equipment. It can cause additional losses in electrical equipment and conductors of power lines of network organizations, and also affect the elements of the controls. The electric power quality that goes beyond the requirements of the Russian State Standard GOST 32144-2013 often has financial consequences (losses) for enterprises with electrical installations.

It should be noted that about $90 \%$ of distortions of electric power quality are a consequence of the operation of electrical receivers of enterprises [2]. In Russia in most cases, technical problems associated with the operation of electrical receivers in violation of the electric power quality are caused by voltage dips or interruptions $(52 \%)$, and higher harmonics of current $(18 \%)$.
The studies carried out in the USA on the statistics of equipment failures under operating conditions with distorted electric power quality showed that $48 \%$ of failures are caused by voltage dips and surges, $22 \%$ by harmonic components; $15 \%$ by voltage unbalance $[3,4]$.

In the countries of the European Union, the main reasons for equipment failure from a decrease in the electric power quality are voltage dips $(23.6 \%)$; shortterm power outages $(18.8 \%)$; prolonged power outages $(12.5 \%)$; harmonic components $(5.4 \%)$; voltage surges (29\%) [5].

Consider the levels of harmonic distortions introduced into the network by the largest industrial power receivers [6-9].

Electric arc furnaces (EAF) during their operation create non-sinusoidal voltages, which depend on the furnace supply voltage. For EAF receiving power supply at a voltage of $6-10 \mathrm{kV}$, the total harmonic component of voltage is $0.5-5.0 \%$, for a $35 \mathrm{kV}$ furnace supply voltage it is $0.7-4.0 \%$ and for a $110 \mathrm{kV}$ supply voltage it is 0.1 $2.0 \%$.

The level of harmonic components generated by rolling mills depends on the type of mill. The average voltage harmonic component for hot plate mills are from 4 to $14 \%$. Blooming and slabbing have a voltage harmonic ratio of 10 to $30 \%$.

In welding networks, the harmonic voltage components depend on the type of electric welding machines and are in the range from 4.2 to $6.0 \%$ [10].

The presented data show that dips and non-sinusoidal supply voltage are the main disturbances affecting the reliable and adequate operation of equipment and automatic control devices of the industrial electrical networks.

\footnotetext{
* Corresponding author: $\underline{\text { sevosaa } @ \text { gmail.com }}$
} 
During processing discrete signals of currents and voltages of utility frequency by conversion units of modern automatic control devices, it is necessary to reduce the sampling frequency. This need may arise, for example, when exchanging data between two digital signal processing devices operating at different clock frequencies. Reduction of the sampling frequency also enables to simplify the hardware and software implementation of individual devices.

At the same time, during digital processing of signals of currents and voltages with the presence of harmonic high-frequency components, with a decrease in the sampling frequency, the processing result may be erroneous and not correspond to the original signal. This situation does not take place when processing currents and voltages that do not contain higher harmonics.

This paper aims at assessing the results of modelling the effect of digital processing of signals of currents and voltages of utility frequency with the presence of distortions in the network in the form of higher harmonics, which occurs when the sampling frequency is reduced and affects the adequacy (coherence) of the obtained results.

\section{Materials and methods}

The sampling frequency can be reduced or increased. Downsampling is known as decimation or compression of discrete samples. Currently, the term decimation is used, as a rule, to mean downsampling by any integer number of times. The upsampling process is known as interpolation, i.e. obtaining estimates of intermediate samples. The elements providing the signal decimation procedure are referred to as downstream discrete systems $[11,12]$.

During digital processing of signals containing harmonic components with a frequency higher than 50 $\mathrm{Hz}$, the implementation of the decimation process can lead to the loss of reliable information about the signal and, as a result, to errors in the operation of automatic control devices. The decimation process has been sufficiently studied in $[11,13,14]$. This paper discusses the influence of downsampling on the result of processing power frequency signals in the presence of higher harmonic components.

The adequacy of digital signal processing is estimated using a discrete correlation coefficient characterizing the distortion of the sinusoidality of the analyzed signal, as well as violation of the coherence requirements [15-17].

In the probabilistic approach, the degree of coherence of two centered random variables $X$ and $Y$ (with zero mean value) is determined as their correlation coefficient $\rho:$

$$
\rho=m\left\{X Y^{*}\right\} /\left[m\left\{|X|^{2}\right\} m\left\{|Y|^{2}\right\}\right]^{1 / 2}=R_{X Y} /\left[R_{X X} R_{Y Y}\right]^{1 / 2},(1)
$$

where $\mathrm{m}\{\ldots\}$ is the mathematical expectation.

For example,

$$
R_{X Y}=m\left\{X Y^{*}\right\}=\iint_{-\infty}^{+\infty} x y^{*} p_{x y}(x, y) d x d y,
$$

where $p_{x y}$ is the joint probability density for the random variables $X$ and $Y$. It is known that for the correlation coefficient the inequality $0 \leq|\rho| \leq 1$ is true. Variables $X$ and $Y$ before applying expression (2) must be centered by subtracting from each value their corresponding nonzero mathematical expectations $m\{X\}$ and $m\{Y\}$. In expression (1), $R_{X Y}$ is the covariance of variables $X$ and $Y$.

In practical calculations the following relations are used for sets of values $X(s)$ and $Y(s)$, where $s$ is the variable characterizing the index of a random value in the set:

$$
|\rho|=\left|R_{X Y}\right| /\left[R_{X X} R_{Y Y}\right]^{1 / 2},
$$

where

$$
R_{X Y}=(1 / N) \sum_{s=1}^{N} \quad X(s) Y^{*}(s) .
$$

It can be shown that if $X(s)$ and $Y(s)$ are selected from the set specified by $p_{x y}$, then the estimated value $R_{X Y}$ (4) tends to $R_{X Y}(2)$ in the probabilistic sense for $\mathrm{N} \rightarrow \infty$.

The degree of coherence is a measure of how closely $X$ and $Y$ are related by a linear transformation. If $X$ and $Y$ are directly related by a linear transformation, then their degree of coherence is close to one. A pair of random variables $X$ and $Y$ is assumed to be absolutely coherent when $|\rho|=1$, and absolutely incoherent when $|\rho|=0$.

To determine the degree of coherence of digital signal processing during downsampling, we use expression (3) for the cross-correlation coefficient. In this case, violations of the cross-correlation coefficient characterizes signal distortions and the presence of errors in the measuring devices of automation and control systems. The distorted signals are compared with the basic ones, as which we accept the signals at the input and output of the decimator when considering the corresponding measuring element of the automation systems.

\section{Results}

To analyze the adequacy of the implementation of digital signal processing, we analytically set the voltage oscillograms, taking into account the influence of distorting factors in the form of voltage harmonics on the fundamental frequency harmonic.

When calculating the correlation coefficients, we accept discrete sinusoidal signals: initial $x(n)=U \cdot \cos \left(2 \pi f_{0} n T_{\mathrm{d}}+\varphi\right)$ and decimated $y(k)=U \cdot \cos$ $\left(8 \pi f_{0} n T_{\mathrm{d}}+\varphi\right)$. In expressions for basic signals, the following parameters are accepted: $U=100 \mathrm{~V} ; f_{0}=50 \mathrm{~Hz}$; $T_{\mathrm{d}}=1 /\left(f_{0} \cdot N\right) \mathrm{s} ; N=80 ; \varphi=0 \mathrm{rad}$.

Further we add to the original and decimated signals the 21 st harmonic at a frequency of $f=42 \pi f_{0}$ with an amplitude of $15 \mathrm{~V}$ and estimate its distorting effect for two signals (Figure 1). We take the initial phase of the distorting harmonic equal to zero. 


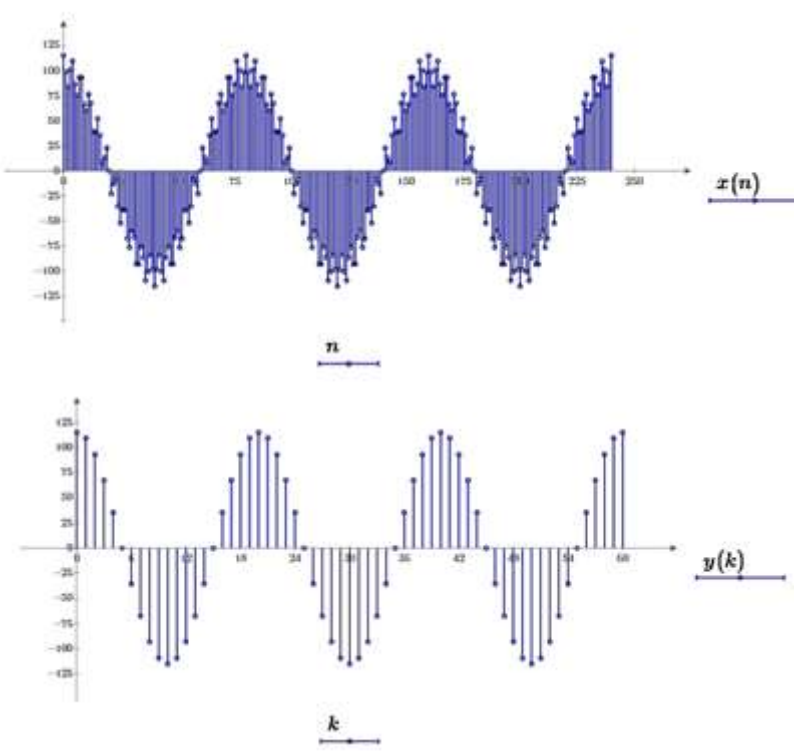

Fig. 1. Oscillograms of a sinusoidal signal distorted by the 21 st harmonic, before and after decimation.

We illustrate the filtering process by the measuring unit of the automation device by calculating the discrete Fourier transform for the power frequency harmonic, and determine the amplitudes of the initial $x(n)$ and decimated $y(k)$ signals (Figure 1) using the expressions

$$
\begin{aligned}
& S x=(2 / N) \sum_{n=0}^{N-1}\left[100 \cdot \cos \left(2 \pi f 0 n \mathrm{~T}_{d}\right)+\right. \\
& \left.+15 \cdot \cos \left(42 \pi f_{0} n T_{d}\right)\right] \cdot \exp \{-j 2 \pi n / N\} \\
& S_{y}=(8 / N) \sum_{k=0}^{(N / 4)-1}\left[100 \cdot \cos \left(2 \pi f_{0} k 4 T_{d}\right)+\right. \\
& \left.+15 \cdot \cos \left(42 \pi f_{0} k 4 T_{d}\right)\right] \cdot \exp \{-j 8 \pi k / N\} .
\end{aligned}
$$

Calculations using expressions (5) and (6) show that the amplitudes of the measured fundamental harmonic of signals $x(n)$ and $y(k)$ (Figure 1) are different and correspond to $\left|S_{x}\right|=100 \mathrm{~V}$, and $\left|S_{y}\right|=115 \mathrm{~V}$. Thus, the discrete Fourier transform (DFT) of the decimated signal did not exclude the $21 \mathrm{st}$ harmonic and caused the distortion of measurement results.

It should be noted that when performing such processing at facilities that use the IEC 61850 standard, as a rule, analog signal measuring converters are used. They are utilized as a source of instantaneous values of currents and voltages for devices that solve problems of control, telemechanics, and quality control of electrical energy. Thus, not only the 21 st, but also other harmonics (41st harmonic with similar parameters (Figure 2)) will be passed by the input circuits of the analog signal converter and will be perceived by the measuring element of the digital device for the decimated signal $y(k)$ as harmonic of the fundamental frequency. Note that the discrete signals $y(k)$ shown in Figures 1,2 are identical under different distorting influences, and the oscillograms themselves do not reflect the effect of this influence.

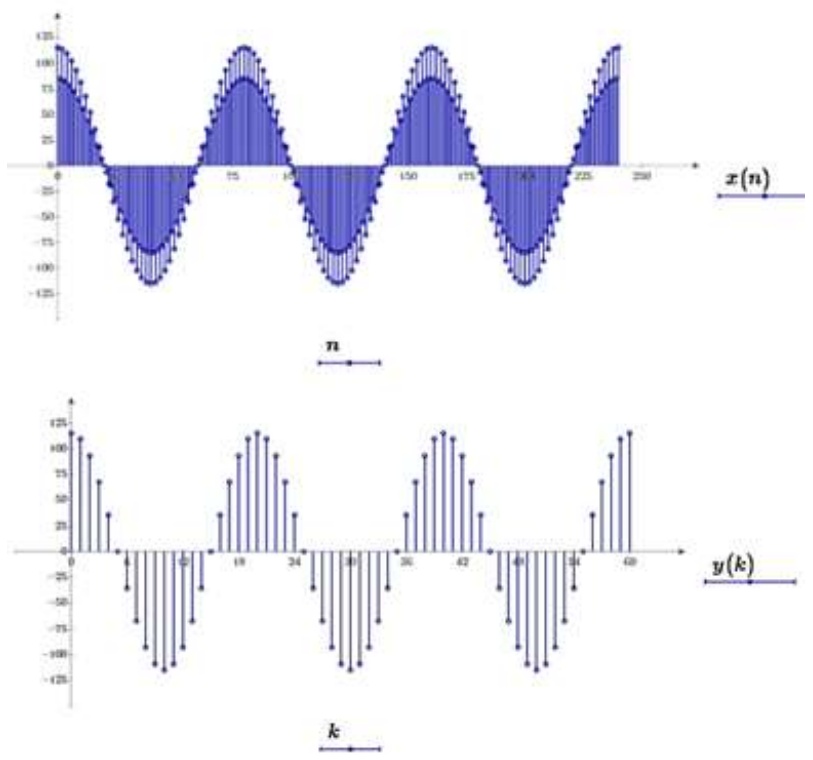

Fig. 2. Oscillograms of a sinusoidal signal distorted by the 42nd harmonic, before and after decimation.

To ensure the coherence of digital processing of $y(k)$ signal taking into account decimation of samples, preliminary digital filtering is required. Such filtering should be carried out for the signal $x(n)$ corresponding to a high sampling frequency, and in terms of computational complexity it is equivalent to the DFT (5). In this case, the decimation operation itself loses its meaning, and it is advisable to evaluate the parameters of currents and voltages for the signal $x(n)$.

It should be noted that the decimation procedure and the subsequent DFT of the decimated signal for devices supporting the IEC 61850 protocol results only in incoherence of digital signal processing and unnecessary computational load.

The influence of interharmonics on the decimation process was studied by introducing a distorting voltage signal with an amplitude of $15 \%$ of the fundamental harmonic amplitude and having a frequency $f=39 \pi f_{0}$. An example of the original and decimated signals is shown in Figure 3.

Analysis of the discrete Fourier transform of voltage signals (Figure 3 ) showed that the interharmonic has the same distorting effect on both signals $x(n)$ and $y(k)$, violating their coherence.

Note that comparison of the oscillograms of signals $x(n)$ and $y(k)$ (Figures 1-3) illustrates the effect typical for operating power grid enterprises and manifests itself when identifying the causes of emergencies. The distortion of the sinusoidality of the signal $x(n)$ by the higher (inter-) harmonic on the oscillogram is clearly seen, but the $y(k)$ signal distortion is practically not detected visually. This circumstance can lead to an incorrect interpretation of the reasons for the operation of automation devices and subsequent incorrect management decisions. Thus, poor visibility of oscillograms is an additional disadvantage of the decimation effect.

Perform a numerical analysis of violations of the coherence of voltage signals by comparing distorted 
signals with harmonic voltage fluctuations at the utility frequency of $50 \mathrm{~Hz}$.

The sequence of calculations is illustrated by the example of processing a distorted signal $x(n)$ at the input of the decimation device. In this case, the expressions for calculating the cross-correlation coefficient take the form

$$
\left|\rho_{x}\right|=\left|R x_{b}\right| /\left[R x x R x_{b} x_{b}\right]^{1 / 2},
$$

where

$$
\begin{aligned}
& \operatorname{Rxx}_{b}=(1 / N) \sum_{s=1}^{N} x(n) x_{b}(n)= \\
& =(1 / N) \sum_{s=1}^{N} x(n) \cdot U \cdot \cos \left(2 \pi f_{0} n T_{d}+\varphi\right),
\end{aligned}
$$

$x_{b}(n)$ are sampled discrete sine wave samples $\left(f_{0}=50 \mathrm{~Hz}\right)$, $\varphi=0 \mathrm{rad}$.

A comparative analysis of expressions (5) and (8) shows that the difference between the estimate of the complex amplitude $S x$ and the unnormalized crosscorrelation coefficient $R_{x} x_{b}$ consists only in the use of complex values in (5). Thus, the similarity of the analyzed expressions once again confirms the possibility of using the cross-correlation coefficient to characterize the coherence of digital signal processing. It can also be used as an indicator of the error rates of the measuring elements of power frequency signals. Note that the autocorrelation coefficients $R x x$ and $R x_{b} x_{b}$ correspond to the energies of the voltage signals $x(n)$ and $x_{b}(n)$, which include $N$ discrete samples.

Similar calculated relations can be formed for signals $y(n)$ and $y_{b}(n)$.

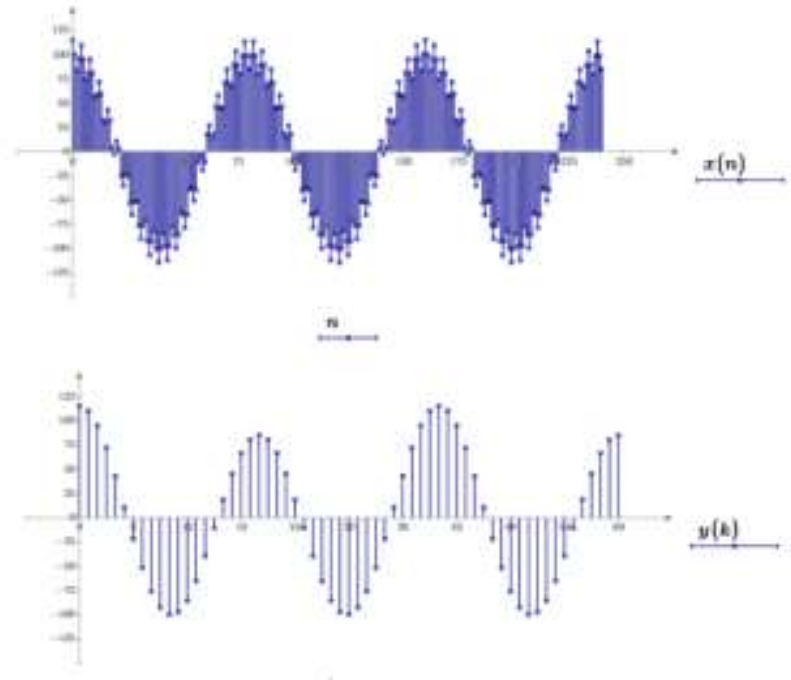

Fig. 3. Oscillograms of a sinusoidal signal distorted by an interharmonic at a frequency of $f=39 \pi f_{0}$, before and after the decimation.
Table 1. Calculated values of the normalized cross-correlation coefficients.

\begin{tabular}{|c|c|c|c|}
\hline \multirow{2}{*}{ No. } & $\begin{array}{c}\text { Variant of voltage signal } \\
\text { distortion by higher harmonics }\end{array}$ & \multicolumn{2}{|c|}{$\begin{array}{c}\text { Normalized } \\
\text { cross-correlation } \\
\text { coefficient }\end{array}$} \\
\cline { 3 - 4 } 1 & $\left|\rho_{x}\right|$ & $\left|\rho_{y}\right|$ \\
\hline & $\begin{array}{c}\text { Voltage signal distorted by the } \\
21^{\text {st }} \text { harmonic, corresponding to } \\
\text { Figure } 1\end{array}$ & 0.989 & 1.00 \\
\hline 2 & $\begin{array}{c}\text { Voltage signal distorted by the } \\
21^{\text {st }} \text { harmonic, having the initial } \\
\text { phase } \varphi=\pi / 4\end{array}$ & 0.989 & 0.995 \\
\hline 3 & $\begin{array}{c}\text { Voltage signal distorted by the } \\
42^{\text {nd }} \text { harmonic, corresponding } \\
\text { to Figure 2 }\end{array}$ & 0.989 & 1.00 \\
\hline 4 & $\begin{array}{c}\text { Voltage signal distorted by the } \\
\text { inter-harmonics, corresponding } \\
\text { to Figure 3 }\end{array}$ & 0.989 & 0.989 \\
\hline
\end{tabular}

Table 1 shows the results of calculating the normalized cross-correlation coefficient for the decimation variants considered above.

\section{Discussion}

Calculations performed according to options 1 and 3 (Table 1) show that the digital processing of the decimated signal violates the coherence requirements. The normalized cross-correlation coefficient between distorted and undistorted decimated power frequency voltage signals is 1 (i.e. the signals are similar), and filtering using the discrete Fourier transform does not eliminate the distorting effect of the $21 \mathrm{st} / 42$ nd harmonic.

Comparison of options 1 and 2 (Table 1) confirms that the nature of coherence violations (distortions) depends not only on the energy characteristics of the distortion (the amplitude of the 21 st harmonic), but also on the initial phase of the harmonic.

The voltage distortion of the interharmonic component for the signals at the input and output of the decimator is equivalent.

Figures 1-3 show that the violation of the coherence of digital signal processing occurs if the spectrum of the decimated signal contains frequencies exceeding half the sampling frequency (in accordance with the Kotelnikov theorem). Their appearance will lead to the presence of false frequency components in the spectrum of the decimator output signal. To eliminate the distorting effect (as during sampling of an analog signal), it is necessary to perform preliminary low-pass filtering with a cutoff frequency equal to the Kotelnikov frequency.

\section{Conclusions}

The development of the electric power industry in the context of the introduction of modern means of intellectualization into the structure of power supply systems is impossible without analyzing the factors that affect the form of currents and voltages when determining the parameters of the electric network mode. 
Digital processing of signals of currents and voltages of utility frequency during decimation must be carried out taking into account the observance of the coherence requirements. Failure to comply with these requirements leads to significant errors in the estimates of the electrical signal parameters.

The cross-correlation coefficient can be chosen as a numerical characteristic that allows one to estimate the distortion of power-frequency signals and characterize violations of the coherence requirements of digital signal processing.

\section{References}

1. G.Ya. Vagin, A.L. Kulikov, The electric power quality in power supply systems, Analysis of the state of the regulation and control methods, Electric stations, 6, 54-59 (2019)

2. L.I. Kovernikova, V.V. Sudnova, R.G. Shamonov, et all, edited by N.I. Voropai, The electric power quality: current state, problems and proposals for their solution (Novosibirsk: Nauka, 219, 2017)

3. S. McNulty, P. \& B. Howe, Electric power quality problems and renewable energy solutions, Submitted to Massachusetts Renewable Energy Trust (September 2002)

4. C.J. Melhorn, A. Maitra, W. Sunderman, M. Waclawiak, A. Sundaram, Distribution system electric power quality assessment phase-II: voltage sag and interruption analysis, Copyright IEEE, PCIC-2005-13 (2005)

5. P. Johnson, R. Coney, Introducing national quality of supply (power quality) standards the South African experience, 10th International Conference on Electromagnetic Compatibility, Conference, 4450 IEE 1997 (September 1997)

6. B.P. Borisov, G.Ya. Vagin, Power supply of electrotechnological installations: monograph (Kiev: Naukova Dumka, 148, 1985)

7. I.V. Zhezhelenko, A.K. Shidlovsky, G.G. Pivnyak, et all, Electromagnetic compatibility of consumers: monograph (Moscow: Mashinostroenie, 351, 2012)

8. V. Zatsepina, E. Zatsepin, O. Shachnev, A. Shachnev, T. Petrov, Analysis of power quality in presence of frequency distortions, E3S Web of Conferences, 178, 01019 (2020) DOI: 10.1051/e3sconf/202017801019

9. N.R. Rakhmanov, V.G. Kurbatsky, H.B. Guliyev, N.V. Tomin, R.N. Rakhmanov, Analysis and modeling of harmonic distortions in consumer's distribution networks, containing a powerful nonlinear load, E3S Web of Conferences, 58, 03015 (2018) DOI: 10.1051/e3sconf/20185803015

10. G.Ya. Vagin, Modes of electric welding machines: monograph (Moscow: Energoatomizdat, 192, 1985)
11. A.B. Sergienko, Digital signal processing: textbook (Saint-Petersburg: BHV-Petersburg, 3rd ed., 768, 2011)

12. L.M. Goldenberg, B.D. Matyushkin, M.N. Polyak, Digital signal processing: Handbook (Moscow: Radio and communication, 312, 1985)

13. P.F. Ribeiro, K.A. Duque, P.M. da Silveira, A.S. Serqueira, Signal processing in smart grids of power systems (Moscow: Technosphere, 417, 2020)

14. L. Rabiner, B. Gold, Theory and application of digital signal processing (Prentice Hall, 762, 1975)

15. C. Cook, M. Bernfeld, Radar Signals: An Introduction to Theory and Application (1967)

16. Ya.D. Shirman, S.T. Bagdasaryan, A.S. Malyarenko, et all, Radio-electronic systems: Basics of construction and theory: Handbook (Moscow: Radiotekhnika, $2^{\text {nd }}$ Ed. rev. and added., 512, 2007)

17. D. Borkowski, Estimation of power system spectral parameters with coherent resampling, Doctor's Thesis, AGH-Univ. Sci. Technol., (Cracow, Poland, 2007) ISBN: 83-918624-7-X.. 